\title{
Sentinel nodes: a promise half fulfilled
}

\author{
John Buscombe $\cdot$ Ziauddin Saad
}

Received: 30 January 2015/Accepted: 3 February 2015/Published online: 19 February 2015

(C) Italian Association of Nuclear Medicine and Molecular Imaging 2015

Scintigraphic localisation of sentinel nodes was first developed over 20 years ago and has been used widely in breast cancer and melanoma for most of the past two decades. On the surface the principle seems so plain and simple it is mystifying why it is not universally understood and accepted. Any tumour, in particular those of ectodermal origin, will have a logical lymph drainage. Any cancer cells that arise in the primary tumour and diffuse via the lymphatic system will use this logical method of spread. Therefore any lymph node on that path, in particular the first such node, will contain cancer cells before they can spread further. This first draining node (or more realistically adjacent nodes) acts as a guardsman protecting the body, thus the use of the word sentinel, the American term for guardsman. The theory underpinning the sentinel node hypothesis is that if this node can be visualized, it can be removed and assessed histologically. The absence of any cancer cells within the sentinel node means it is unlikely that cancer cells could have spread further through the lymphatic system and no further removal of tissue is warranted. Conversely, if there are tumour cells within the sentinel node there is a chance that the cancer has spread further and appropriate treatment can be initiated. An alternative method would be to remove all the lymph nodes from the lymph node basin draining a particular cancer (e.g., a full axillary dissection if the patient had a breast cancer). In certain areas such as the axilla, this degree of lymph node removal can lead to marked morbidity such as

In Memoriam:Donald Morton 1934-2014.

J. Buscombe $(\varangle) \cdot$ Z. Saad

Nuclear Medicine, Cambridge University Hospitals,

Cambridge, UK

e-mail: jrb.wjnm@googlemail.com nerve damage and lymphoedema of the arm. Even when lymphoedema is not the issue there may still be significant morbidity related to extensive lymph node dissection, for example in head and neck cancers, as well as significant guesswork as to which nodes to remove. Therefore in melanoma, head and neck cancers, and cancers of the penis, vulva and cervix sentinel techniques may not just reduce surgical morbidity but increase the probability of finding a node which actually drains the cancer primary.

The sentinel node technique is effectively a staging technique and as such should only be used in those patients in whom no nodal or metastatic disease is found clinically or by other means such as imaging. Thus in terms of TNM staging it should only be used in TxNOM0 cancers.

The situation with melanoma can be very different than in breast cancer, in that the primary has normally been removed at the time of diagnosis. This can result in changes in lymphatic drainage that may mean the node identified is not the sentinel node. It has therefore become normal practice to use multiple injections around the excision site to ensure that the true sentinel node is not missed. This often means that multiple sentinel nodes are identified and on the trunk not only are there multiple nodes but there may be multiple draining basins. This may result in extensive surgery to identify and remove all these nodes, though for some the multiple basins can be a negative prognostic factor. In addition, unlike breast cancer there are limited options if the disease has become metastatic. This has led observers to question the fundamental basis for sentinel node localisation in melanoma, and the debate has moved from the medical press to a more public domain [1]. The argument is that despite a large USbased trial (multicentre selective lymphectomy trial MTSL-1) there is as yet no clear evidence that sentinel node identification and removal changes outcomes for the 
patient and effectively this procedure constitutes overtreatment which could offer false hope to patients and result in high expenditure, including the cost of the nuclear medicine procedures. This was a provocative statement to all those involved in the practice of sentinel node localisation. This is coupled to the problem of access to this technique, where uptake of the methodology can be patchy. In those countries with highly centralised health systems using hub and spoke cancer centres this problem is not so great, but where services are not so organised the institution doing the surgery may not have access to nuclear medicine, especially modern techniques such as SPECTCT.

However, the argument may come down to semantics and the view taken by different practitioners working in melanoma. For the surgeon, a lower morbidity operation that produced the same result in terms of success may be considered appropriate. This may apply, for example, in the results of MTSL-1, which reviewed the surgical outcomes in 1,269 patients with intermediate thickness $(1.2-3.5 \mathrm{~mm})$ melanoma [2]. All the patients randomised were clinically and radiologically without nodal or metastatic disease. Patients were randomised to either observation only or to sentinel node biopsy and, if this node was found to be positive, subsequent block dissection of the draining basin. Results showed the 5-year disease-free survival rate to be significantly greater for sentinel node biopsy $(78 \%)$ than for observation $(73 \%)$. The biggest difference, however, was in local nodal recurrence, the rate being $16 \%$ in the observation group vs $3 \%$ in the sentinel node group. To a surgeon who would have to operate and clear local nodal recurrence, the pay-off between the low-morbidity sentinel node study and the high-morbidity reoperation with recurrence would be an acceptable outcome and as such sentinel node localisation can be seen as successful. Looking at the process from an oncologist's point of view, however, the sentinel node biopsy has no effect on 5-year overall survival or even melanoma-specific deaths, though a positive sentinel node was a predictor of likely melanoma-related death within 5 years.

The debate has been moved forward by the final report of MTLS-1, the study now including 2,001 randomised patients. There is now a reported significant improvement in melanoma-specific survival rates to $71 \%$ in those who had sentinel node biopsy, compared to $65 \%$ in those with observation only, although it was noted this difference was reduced in those patients with thicker primary tumours, probably because any advantage of sentinel node biopsy was negated by the high-risk tumour [3]. Therefore we have the possibility that sentinel node biopsy has a therapeutic as well as a diagnostic role and may indeed result not only in improved staging and reduced surgical morbidity but also in improved patient outcomes.

The sentinel node technique is now expanding into different clinical scenarios with multi-centre trials in head and neck cancers in both the USA and Europe that should report during the coming 1-2 years. These offer hope that patients with oral cancers will no longer need to suffer the consequences of widespread cervical lymph node block dissection, often called a "commando operation".

Therefore in many ways sentinel node scintigraphy is in its "teenage" years. It is troublesome in that the technique is not mature enough to have yielded long-term outcome data, but it is a technique that demands our attention and cannot be ignored. For those in the nuclear medicine community it is indeed a true embodiment of the "tracer technique". Though SPECT-CT and hand-held imaging devices enhance the quality of images produced, the underlying principles can be realised in most nuclear medicine centres, and with the availability of low-cost intraoperative probes there is no reason these techniques cannot be universally available to benefit patients worldwide.

Conflict of interest Prof. John Buscombe is a paid consultant of Navidea. Dr. Ziauddin Zia Saad has no conflicts of interest.

\section{References}

1. Torjessen I (2013) http://www.bmj.com/content/346/bmj.e8645. Accessed 14/12/14

2. Morton DL, Thompson JF, Cochran AJ et al (2006) Sentinel-node biopsy or nodal observation in melanoma. New Engl J Med 355:1307-1317

3. Morton DL, Thompson JF, Cochran AJ et al (2014) Final trial report of sentinel-node biopsy versus nodal observation in melanoma. New Engl J Med 370:599-609 